\title{
El dilema de la ciencia, la política y la simulación desde el pensamiento complejo
}

\section{The dilemma of science, politics, and simulation from complex thinking}

\author{
Adriana Marcela Cabezas Moral
}

(.)

Recepción: 01/10/2020
(.)

Aprobación: 09/11/2020
-

Publicación: 18/12/2020

Para citar este artículo:

Cabezas Mora, A. M. (2020). El dilema de la ciencia, la política y la simulación desde el pensamiento complejo. Indagare, (8), 260-265. https://doi.org/10.35707/indagare/825

\section{(9) $(1 \Theta \Theta$}

${ }^{1}$ Politóloga. Grupo de investigación MYSCO, Universidad de Ibagué, Colombia. Correo electrónico: adriana.cabezas@ unibague.edu.co 


\section{Resumen}

El siguiente texto contiene la entrevista realizada al profesor e investigador argentino Leonardo Rodríguez Zoya. Se presentan dos momentos alrededor de la experiencia investigativa y personal del invitado: por un lado, el proceso de encuentro con el pensamiento complejo como su punto de llegada, y por el otro, la experiencia de la simulación compleja en la política pública argentina mediante el modelo SocLab. Esta reflexión tendrá como invitación final la continuidad de los estudios de la complejidad como una apuesta para comprender la crisis de época actual y el papel de las instituciones educativas en adelante.

\section{Abstract}

The following text contains the interview with the Argentine professor and researcher Leonardo Rodríguez Zoya. Two moments are presented around the research and personal experience of the guest: on the one hand, the process of encountering complex thought as its arrival point; and on the other, the experience of complex simulation in Argentine public policy through the SocLab model. This reflection will have as a final invitation the continuity of the studies of complexity as a bet to understand the current crisis and the role of educational institutions from now on.

Palabras claves: Pensamiento complejo, simulación, política pública, ciencia.

Key words: Complex thinking, simulation, public policy, science.

Figura 1. Retrato de Leonardo Rodríguez Zoya

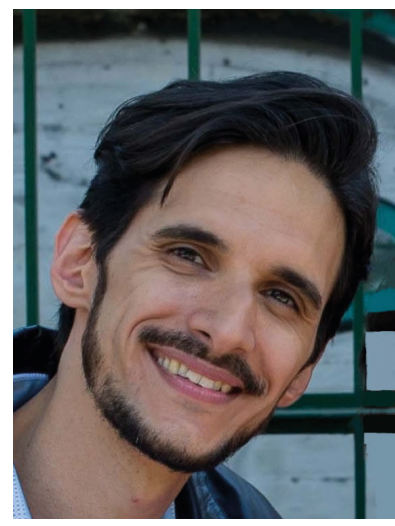

Fuente: Suministrada por el investigador 


\section{Introducción}

En el marco del auge y agudización de problemáticas políticas y sociales tras la irrupción de la pandemia de la COVID-19 a nivel mundial, y la puesta en escena de grupos e institutos dedicados a la investigación y la reflexión desde el pensamiento complejo y sistémico, Leonardo Rodríguez Zoya comparte su experiencia académica investigativa a partir de dos momentos: el punto de partida para su inmersión en temáticas de la complejidad y desde su experiencia en el proyecto investigativo SocLab como ejemplo exitoso de la simulación sistémica.

Rodríguez es profesor e investigador, es doctor en Sociología por la Universidad de Toulouse 1-Capitole, Francia y doctor en Ciencias Sociales por la Universidad de Buenos Aires (UBA), ha participado en diversos eventos académicos en el plano internacional y actualmente es docente en la UBA. Leonardo participó como investigador internacional invitado en los Cursos de Verano de la Universidad de Ibagué desarrollados en el semestre A-2002 con el curso Ciencia, política y futuro: un análisis desde la complejidad. A la vez, existen lazos fraternos debido al trabajo investigativo del profesor con el Grupo de Investigación de Modelado y Simulación de Sistemas Sociales Complejos (MYSCO) adscrito a la misma institución educativa.

\section{El punto de llegada a la complejidad como un proyecto de vida}

Rodríguez Zoya cuenta con una amplia formación académica que le ha permitido introducirse en diversas disciplinas y áreas de interés: la ciencia política, la sociología, la informática, las artes, la poesía, etc.; hecho que cultivó su interés en analizar los fenómenos sociales desde una mirada holística y transdisciplinar. Es fundador y director de la Comunidad de Pensamiento Complejo ( $\mathrm{CPC}$ ), una de las más importantes de América Latina en este ámbito, su arribo a temáticas de la complejidad viene marcado por pasajes recónditos en su vida.

En el proceso de formación como politólogo en la UBA, disciplina que eligió debido a su interés en conocer y ejercer en el mundo de la política, aunque también soñaba con ser actor y poeta, se encontraría con una de las discusiones más sobresalientes de la ciencia política como lo es el estatuto científico de la política como ciencia. Disyunción expresada de la siguiente manera: la ciencia al servicio del interés político y la política como un mero instrumento de la primera.

En adelante esta ruptura calará hondo en las aspiraciones académicas de Leonardo, quien se interesará en los estudios inter y transdisciplinares para dar cuenta de los diversos y complejos fenómenos sociales. A la vez, es evidente la influencia del pensador francés Edgar Morin, figura destacada de los estudios de la complejidad a 
nivel mundial, y quien será el maestro de Leonardo, previo y posterior a sumergirse en el pensamiento complejo y sistémico. La obra de Morin representa entonces una conmoción académica e intelectual para Leonardo que definirá la complejidad como el eje central de su proyecto de vida.

De hecho, una de las experiencias más preciadas por el profesor Rodríguez fue compartir el proyecto investigativo sobre simulación que venía desarrollando con un colega de la licenciatura en ciencia política ante Edgar Morin en un Congreso académico en La Habana, Cuba. Encontrarse ante la presencia de esta figura académica prestigiosa y el interés de adentrarse más en su obra le condujo a estudiar francés para comprenderle mejor, y, a la vez, hallar una posibilidad de continuar estudios de posgrado en el extranjero. Dos acontecimientos surten de sus estudios en la lengua francesa: es aceptado como estudiante de posgrado en la modalidad de cotutela en la Universidad de Toulouse en Francia y conoce a quien es hoy su pareja sentimental. De forma que el resuelto interés de Leonardo en adentrarse a Morin y la complejidad moldea su vida académica y personal.

El interés del trabajo en equipo y estrechos lazos de amistad han sido característicos en su trayectoria investigativa. En compañía de colegas y amigos nace en 2010 CPC, que hoy posee miembros de diversos puntos geográficos y alberga un repositorio bibliográfico de carácter abierto para la comunidad (RedCOM, s.f.). La CPC es una red de conocimiento gratuita que promueve un estilo de pensamiento complejo entre las ciencias, la política, la cultura y la educación. El objetivo es que esta comunidad se expanda cada vez más para promover una reflexión sistémica amplia.

\section{El modelo SocLab como posibilidad de incidir en la política pública}

De la amplia experiencia investigativa de Leonardo se destaca el modelo SocLab concentrado en un clúster de frutas finas en la región de la Patagonia argentina, específicamente en la región del Neuquén y la Comarca. Bajo el plan denominado de mejora competitiva se ha venido desarrollado el comercio de diversas frutas artesanales por parte del Ministerio de Agricultura de Argentina.

A nivel macro con el SocLab se retó por comprender cuál sería la posibilidad de que productores heterogéneos y con herramientas tecnológicas desiguales pudieran apostar por construir colectivamente un clúster que fuere benéfico para todos. La región del Neuquén y la Comarca es especial debido a las condiciones diferenciales de cosmovisiones, idiosincrasias e intereses de los agricultores. Dos etapas caracterizan el desarrollo del proyecto: la investigación cualitativa y el modelo computacional desarrollados de 2013 a 2017. El trabajo fue muy dedicado en la apuesta por la 
comprensión sistémica del fenómeno, razón por la cual desde diversos observadores se lograron identificar los actores involucrados, los recursos y las relaciones que se presentan entre ellos mismos en las diversas etapas del proceso.

En la comprensión de este juego social, por medio del modelo computacional, se logró determinar (medir) la capacidad de influencia de los actores, desde los agricultores, los intermediarios, la cadena comercial y el ente gubernamental en la toma de decisiones, y se halló que este proceso se concentra en el Ministerio, la coordinación del clúster y, en menor medida, en los productores.

A la larga la intención del SocLab y de otros proyectos en los cuales el profesor Rodríguez ha venido trabajando es la de comprender las relaciones de poder, efecto y solidaridades presentes en eventos sociales, económicos y políticos, cuyo objetivo principal es contribuir en la construcción de políticas públicas exitosas. Bien es sabido que tradicionalmente las políticas públicas en Argentina y Latinoamérica en general se realizan de espaldas a las cosmovisiones y sentipensares de las comunidades involucradas, hecho que explica en buena medida el fracaso de las mismas. De forma que la apuesta del SocLab es la de comprender y resituar el lugar de la comunidad en dichos procesos, dado que en últimas la política debe estar al servicio de los que ya la necesitan, no al contrario.

A pesar de la enorme expectativa que tuvo el proyecto y el equipo de trabajo, se encontró que cuando ya habían avanzado lo suficiente en el modelo, el clúster de frutas ya estaba avanzando y fracasó pronto debido a la compleja relación comercial entre actores heterogéneos tal como lo predijo el modelo. Aun así, el profesor Leonardo y su equipo multidisciplinario continúa trabajando, a sabiendas de que la praxis política suele ignorar el diagnóstico académico.

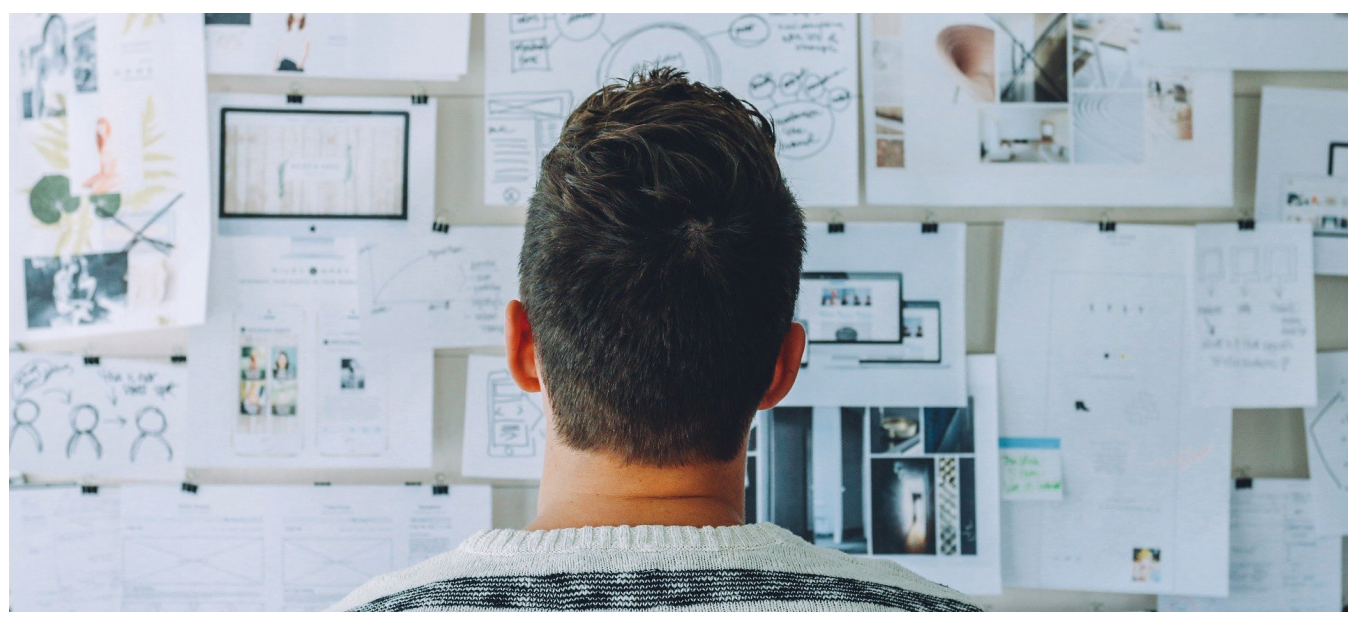


INDAGA3E e-ISSN: 2357-5042 • Número 8 (2020) • Universidad de Ibagué • doi: https://doi.org/10.35707/indagare/825

De otra manera y frente al contexto pandémico presente hoy en el mundo, surte central el papel de la universidad y en general de los establecimientos educativos frente a los procesos formativos del ser humano en todos sus niveles, es decir, desde la educación básica hasta la superior. Sin embargo, no es la única encargada de la promoción de una nueva era de pensadores que despierten al mundo frente a los cambios vertiginosos que se han experimentado en los últimos meses. Se está asistiendo a un escenario de crisis que no es nueva, sino que es el resultado de una serie de contradicciones desde la ciencia, la política, la economía, etc. Frente a esto, Leonardo insiste en que es necesario que las diversas instituciones, empezando por la universidad, converjan a dicho cambio.

La academia tiene hoy una necesidad del uso tecnológico y la implementación de este tipo de herramientas que van a continuar siendo centrales en adelante para los procesos de aprendizaje. No obstante, hay que distinguir que el verdadero reto es acoger la tecnología como un medio más, no como el fin educativo, por lo tanto, a la larga no constituye de por sí un parteaguas frente a como se han venido dinamizando los procesos educativos; la simulación tal como lo considera Leonardo, es claro ejemplo de ello.

En últimas, así como el profesor Leonardo Zoya lo ha experimentado a nivel profesional y personal, el pensamiento complejo brinda herramientas para comprender la crisis actual en sus múltiples dimensiones, como una apuesta a atreverse a pensar sobre los diversos futuros posibles en un mundo en el que nos han arrebatado la posibilidad de pensar de forma diferente. El llamado entonces, por parte del investigador internacional, es a unir las percepciones del ser desde la ética, la praxis, la epistemología y la política en la posibilidad de soñar y diseñar realidades posibles y pluridiversas en las que quepamos todos desde la heterogeneidad; de allí que uno de los lemas de la CPC que encabeza Leonardo sea la religancia, es decir, la insistencia de unir todo aquello que ha sido apartado y desunido. Por eso, su aporte investigativo es de enorme calado para los pensadores que hoy reflexionan desde la complejidad y para quienes lo harán en próximas oportunidades.

\section{Referencias}

RedCOM. (s.f.). Comunidad de Pensamiento Complejo. Recuperado de https://pensamientocomplejo.org/ 